\section{Conference report: Nano tech + Future 2003 Chiba, Japan, February '03}

\author{
M.B. Cortie \\ 1st April 2003 \\ Director \\ Institute for Nanoscale Technology \\ UTS \\ Sydney \\ Australia
}

\section{Introduction}

In late February 2003 the second in what promises to be a highly influential series of exhibitions on nanotechnology was held at the Mukuhari Messe Exhibition and Convention Centre in Chiba, Japan. The first event, held in 2002, is said to have received good support from the Japanese technological and academic sectors. However, this year's event was apparently even bigger with $23,000 \mathrm{~m}^{2}$ of exhibition floor space and 23 overseas country booths, including one from Australia. Besides playing my part manning the Australian booth, I made additional use of the event by presenting a paper at the conference (1), as well as by collecting as much information as I could on the applications of gold in nanotechnology. In addition, I visited Japan's National Institute for Materials Science (NIMS) in Tsukuba Science City, where I gave a talk "The strange physical and chemical properties of gold nano-particles".

Why would anybody use gold in nanotechnological applications? The answer is very simple, its nobility. Under atmospheric conditions almost any other metallic element will be covered by a surface oxide coating with a thickness varying from half a micron to as much as a millimetre. This could be a problem given that many of the proposed and existing nanotechnological systems require metallic components of less than $100 \mathrm{~nm}$ thickness! In many instances such a tiny metallic component would be rapidly consumed by oxidation. One solution is to try to protect the surface of the nanoscale metallic component from oxidation, another, possibly better solution, is just to select a metal that is free from this difficulty. The former strategy has been very successfully applied to components made of silicon, which can be passivated with a monomolecular film of an adsorbant such as hydrogen. This, and the enormous existing industrial infrastructure dedicated to silicon, explains why so much of the activity in nanotechnology involves silicon. On the other hand, it appears that the next most popular materials of construction proposed for nanotechnological devices are gold and DNA. Silicon, gold and DNA offer convenient, but very different, processing possibilities. As a general observation, silicon appears well suited for the 'top-down', lithographic-based techniques already used by the electronic industry, and of course it is a versatile semiconductor. DNA appears not to be an electrical conductor (despite some recent controversy on this point), but has a unique capability to be structured into complex 3-D meso-structures by 'bottom-up' techniques. Gold is highly amenable to both lithographic and 'bottom-up' techniques of fabrication, and has the added advantage that it is especially, perhaps even uniquely, suitable for combination with DNA and protein meso-structures (e.g. 2). 


\section{Nanotechnology + Future 2003}

\subsection{Exhibition}

With over 200 stands, the exhibition hall was large and busy. It was very well attended and the organisers reported that by the end of the week over 25,000 persons had passed through. My observation was that it was a more significant drawcard than the conference itself. My colleagues and I spent at least ten hours, spread over the three days of the event, trawling the exhibition for technical information. A minor problem encountered was that the larger proportion of the posters and written information was in Japanese. However, in most (but not all) cases an individual manning the booth was able to elucidate the content.

A close examination of the materials on display was frequently able to reveal some aspect, even if minor, that involved the use of gold. Use of gold bonding wire, contacts and substrates was not uncommon, however in the present report I will concentrate rather on the specifically 'nanotechnological' uses of gold.

The large NEDO stand ${ }^{1}$ contained posters describing the use of gold electrodes for determining the concentration of organic species in aqueous solution (evidently by electrooxidation but some language difficulties intruded in this respect so I am not absolutely sure) and the well-known use of gold-coated substrates as platforms on which to perform self-assembly of organic molecules.

A collaborative poster by Mitsubishi Materials and Dai Nippon Toryo described the manufacture of gold nano-rods, of $70 \mathrm{~nm}$ length by about $8 \mathrm{~nm}$ diameter. These were described as having potential applications in optical filters and for manipulating infra-red radiation. These particular gold rods have excellent aspect ratios compared to those reported by others.

The SET Tsukuba Research Centre had a poster describing gold deposited onto Ni alloy 'bumps' for packaging chips using 'ultrasonic' flip chip technology. The nickel was deposited by the electroless method, and the gold layer is required to protect the nickel from oxidation. The individual gold-coated 'bumps' were $12 \times 12 \mu \mathrm{m}$ square and $7.5 \mu \mathrm{m}$ deep. Flip-chip technology has the potential to displace gold bonding wire from much or some (opinions differ!) of its previous market niche. The use of gold coatings in flip chip technology is therefore a partial compensation for this change.

A poster of the MIRAI project (a multi-institute initiative which seeks to develop the next generation of electronics, and which lies within Japan's AIST science organization), described the use of gold as the gate electrode in new experimental transistors using high-к dielectrics such as $\mathrm{HfO}_{2}$. The gold is evidently used for reasons of chemical compatibility with the production process.
Japan's AIST had a stand showcasing the best of local research, and there information was presented on a 'correlated electron spin tunnelling device' which, once again, used gold electrodes. Unfortunately the main text was in Japanese and a reference provided on the poster to an English-language scientific paper proved, when followed up, to relate to something completely different.

AIST and Fujitsu Limited also displayed a 'magnetoresistive switching device' made from GaAs and gold nanoclusters. It was said to have the highest magneto-resistance ratio $(10,000 \%)$ ever achieved. The application would ostensibly be as an ultra-sensitive magneto-resistance sensor or as a spin-electronics device.

Tanaka Kikinzoku Kogyo K.K. had samples of gold nanoparticles in colloidal suspension on display, and advertised these materials as being for sale in the 20 and $40 \mathrm{~nm}$ sizes. They also offered $\mathrm{HAuCl}_{4}$, and a variety of PGM colloids.

At the Indian stand, posters described how gold nanoparticles could be printed at very high resolution onto a flexible plastic substrate, using a hydrosol and the dip pen method. Another poster described how lysine-modified gold particles could be attached to DNA molecules, however, no particular application for these technologies was cited. More immediate perhaps, was the use of gold nano-particles for a colorimetric test for the pesticide endosulfan, which was claimed to be sensitive down to levels of $0.1 \mathrm{ppm}$. Endosulfan is an orgonochlorine pesticide linked to serious public health problems in India on account of poorly controlled use (3).

Finally, a stand of Germany's Fraunhofer Institute showcased the 'stacked patch' antennae of Porche's (experimental?) new 'adaptive' cruise control. Evidently, the device operates like an on-road radar, and would bring the vehicle out of cruise control and de-accelerate if it detected an obstacle ahead. The device used nanoscale layers of gold. On being questioned regarding the choice of gold, as opposed to some other conductor, Dr Michael Popall explained that at the high frequencies involved (from 28 to $77 \mathrm{GHz}$ !) the current is carried only on the extreme surface layers of the conductor, necessitating an oxide-free surface. Hence gold. If generally true, this implies that other new applications for gold in analogue circuits should open up as the region of spectrum from $1 \mathrm{GHz}$ to $1 \mathrm{THz}$ is increasingly exploited.

\subsection{Conference}

According to the conference organisers, over 3,500 people registered for the conference. However, there were many parallel sessions, and as usual, attendance tailed off in the

${ }^{1} \mathrm{NEDO}$ is the umbrella Japanese government science agency, and was one of the main sponsoring organizations for the exhibition 
afternoon. Nevertheless, the quality of the presentations was in general very good.

The use of gold as a mask in new generation lithography techniques was mentioned by a Japanese team (4). Most research is focused in ultra-violet lithography, which might use chromium metal in the mask. However these workers used a flux of high energy bromine ions for their lithography, and chose to use a mask of gold, as apparently do many individuals working with $\mathrm{X}$-ray lithography.

When immersed in dielectric matrices, gold nanoparticles can have non-linear optical properties, and this topic has been the focus of a modest amount of research around the world. This theme saw expression at the conference in a paper submitted (5) from Singapore's A*STAR Institute of Microelectronics. The author was supposed to be showing how hybrid core-shell nano-particles comprised of gold and cadmium selenide could be used to obtain very high, third-order, non-linear behaviour. Unfortunately he or she did not pitch. This topic is of interest in the context of optical telecommunication circuitry, for use in optical switches and polymer waveguides, although it must be pointed out that conventional bulk non-linear materials are still completely dominant in current devices.

My own talk (1) was not directly concerned with gold, however, I did mention its occasional use in nano-scale coatings on window glass to selectively block infra-red radiation. Currently, other coating systems, such as composite films of silver and dielectrics, are more popular. The reason for this has more to do with aesthetics than with the intrinsic values of gold and silver - and relate primarily to the consumer's mixed response to golden-hued window glass. Silver-coated glass has a more acceptable grey-blue hue. Even in the case of gold, the cost of the metal used is only a small fraction (less than 5\%) of the total cost of the coating, with the bulk of the cost originating from the high capital and running costs of the vacuum coating plant.

Paul Mulvaney, also from Australia, gave a talk on core-shell nano-particles (6). He has worked for some years on nanoparticles which have a gold core and a silica outer layer (the 'shell'). Such particles can be used to prepare two- and threedimensional photonic crystals, or various kinds of opalescent composites. However, at this stage, no commercial exploitation of these ideas seems to have taken place.

Two talks at the conference, which did not even mention gold, were nevertheless quite thought provoking. The use of nano-particles of FePt was the first example. This material was said to have the highest practicable magnetic coercivity of all magnetic materials, and its use in hard disk storage systems has grown remarkably in the last ten years. Of course very little Pt is used per hard disk and so the high cost of the Pt is readily absorbed. This talk by a Japanese speaker, was 'off the program' and I can supply no formal reference to it. The second example related to semiconducting quantum dots, such as CdSe (7). This speaker claimed that over 6,000 research articles and 1,500 patents had been written which addressed these materials. A gram of CdSe was said to cost a merry US\$2 million, but of course individual batches sold to commercial users contained only a few nano-moles of the substance, so are much more affordable. Once again, these are examples of how the cost of the input raw materials is often insignificant in this young industry, since an individual application does not use much and the benefit is large. The take-home reminder is that gold can also benefit from this paradigm, provided that suitable high-tech industrial applications for it can be found and exploited.

A speaker from Italy's University of Lecce was scheduled to give a talk on the use of DNA and other organic molecules in prototype 'molecular electronics' circuits (8). I was not able to attend this talk, but fortunately the authors had recently published similar work in the literature (9), so details of their research could be gleaned from that. They use gold electrodes as probes to establish electrical connections to molecular electronic devices. The use of gold appears to relate to the need to be able to fabricate very precisely dimensioned conductors using electron lithography. As mentioned previously, the dimensions of the oxide layer on most other conductors would exceed those of the desired device.

A speaker from a Dutch university (10), whose talk I did not attend, apparently spoke about 'bottom-up' nanotechnology in general. Buried in the abstract for his presentation was the point that gold-coated probes offer a very useful way to manipulate large organic molecules into non-covalently bonded nano-scale configurations. As potential applications go this one would probably have a negligible impact on consumption, but it once again illustrates how gold's unique blend of oxidation resistance and thiophilicity may be harnessed in nanotechnology.

\subsection{Research at the National Institute for Materials Science (NIMS)}

The NIMS is Japan's biggest and post prestigious materials science and engineering laboratory. Nanotechnology has been identified at NIMS as being an important emerging field of science. They have allocated US\$11 million to the field in 2002 alone, and are refurbishing and/or constructing $13,000 \mathrm{~m}^{2}$ of lab space for the activity, to be ready in early 2004. The projects at NIMS are very diverse, and it seemed a good place to investigate the links between gold and nanotechnology. I met with several programme leaders and a few of the individual researchers. The following pieces of information may be of interest.

Gold nano-particles can be manipulated individually by a novel probe developed at NIMS, and stacked into towers and patterns. The arrangements can be welded to one another 
by application of an electric current (11). A suggested application for the technology was for electronic contacts (as per flip chip type concept) of next generation chips.

In a rather different line of work, a $20 \mathrm{~nm}$ thick gold layer was applied to the surface of complex composite polymermetal spheres proposed as a material for passive damping of acoustic and vibration energy (12). In this case it appears that it was the corrosion resistance of the gold in combination with its easy deposition which was sought.

Of course, many of the researchers mentioned the use of gold substrates in the production of self-assembled monolayers, and the possibility of using it as conductors in molecular-electronic devices. Interestingly, one of them observed that research into simple spherical gold nanoparticles was now considered 'low tech' and it was the other forms and shapes of gold that were state-of-the-art.

\section{Conclusions}

Gold's chemical and metallurgical properties have guaranteed a role for the element in the emerging field of nanotechnology. It is certainly true that individual applications will consume almost insignificant quantities of gold and, given uncertainties regarding how the field will develop, it seems unwise to try and estimate how much additional consumption of the metal will arise. However, an expanding role for the element seems assured.

In general, I expect that gold will be widely used in the form of nanoscale coatings, as electric conductors on twoand three-dimensional nanoscale devices, and in the form of nano-particles and more exotic shapes, for filtering or modifying electromagnetic radiation.

\section{References}

1 C. Masens, G. Smith and M. Cortie, Nanotechnology products for the energy-efficient home, Nanotech 2003 + Future Congress, Chiba, Japan, 26th-28th February 2003. p.226

2 S. Xiao, F. Liu, A.E. Rosen, J.F. Hainfeld, N.C. Seeman, K. Musier-Forsyth, and R.A. Kiehl, Assembly of nanoparticle arrays by DNA scaffolding, J. Nanoparticle Research, vol. 4, pp. 313-317, 2002

3 http://www.cseindia.org/htm//endosulfan/endosulfan_index.htm, accessed April 2003

$4 \mathrm{~K}$. Awazu et al, 3D-nano fabrication for $\mathrm{TiO}_{2}$ by energetic beams, Nanotech 2003 + Future Congress, Chiba, Japan, 26th-28th February 2003, p.227

5 E.S. Kong, Nanophotonics and optical communication technology, Nanotech 2003 + Future Congress, Chiba, Japan, 26th-28th February 2003, p.222

6 P. Mulvaney, Core-shell nanocrystals - Towards designer materials, Nanotech 2003 + Future Congress, Chiba, Japan, 26th-28th February 2003, p.240

7 V. Biju et al, Synthesis of narrow-disperse luminescent CdSe quantum dots, Nanotech 2003 + Future Congress, Chiba, Japan, 26th-28th February 2003, p.224

8 R. Cingolani, DNA basis and proteins for transistor devices, Nanotech 2003 + Future Congress, Chiba, Japan, 26th-28th February 2003, p.232

9 R. Cingolani et al, Nanotechnology approaches to self-organised biomolecular devices, Physica E, vol.13, 2002, pp.1229-1235

10 D.N. Reinhoudt, What is bottom-up nanotechnology? Nanotech 2003 + Future Congress, Chiba, Japan, 26th-28th February 2003, p.73

11 K. Mikihiko et al, Fabrication of microstructures and microdevices by the particle assemblage, Smart Structure and Materials 2001: Smart Electronics and MEMS, V.K. Varadan (ed.), proc. SPIE, vol. 4334, 2001, pp. 263-264

12 S. Kishimoto and N. Shinya, Mechanical property of metallic closed cellular materials containing organic material for passive damping and energy-absorbing systems, J. of Intelligent Materials and Structures, vol.12, 2001, pp. 271-275

\section{ATTENDING A TECHNICAL CONFERENCE INVOLVING GOLD?}

In keeping with its objective of publishing the latest progress, an aim of Gold Bulletin is to publish reports of relevant conferences that involve the science, technology and applications of gold.

The Technical Editor would like to hear from readers planning to attend such conferences and who are willing to write a conference report for publication. A small honorarium is payable for reports published.

Please contact Dr P.M. Harris (address \& e-mail on contents page 37) 